\title{
Invariant subspace method for fractional Black-Scholes equations
}

\author{
Pisamai Kittipoom \\ Applied Analysis Research Unit, Department of Mathematics, Faculty of Science, \\ Prince of Songkla University, Hat Yai, Songkhla 90112 Thailand \\ e-mail: pisamai.k@psu.ac.th
}

Received 2 Mar 2018

Accepted 2 Dec 2018

\begin{abstract}
In this paper, the invariant subspace method is used to solve the time- and pricing-fractional Black-Scholes equations, in which the fractional derivatives are described in the Caputo and Weyl sense, respectively. We introduce invariant subspaces for time and pricing differential operators. Applying an appropriate invariant subspace will reduce the fractional Black-Scholes equation to a system of ordinary fractional differential equations. Finally, using point symmetries of the obtained system, we construct the explicit solutions of the fractional Black-Scholes equations.
\end{abstract}

KEYWORDS: fractional partial differential equation, analytical solution

MSC2010: 34A08 35R11

\section{INTRODUCTION}

The Black-Scholes model is one of the most wellknown option pricing model for determining the value of European vanilla call and put options. Under the assumption that the asset price satisfies the log-normal diffusion process without transaction costs, the classical Black-Scholes equation provides the price $V(x, t)$ of the put or call options at asset price $x \in \mathbb{R}^{+}$and at time $t \in[0, T]$ of a stock or derivative,

$$
V_{t}+\frac{\sigma^{2}}{2} x^{2} V_{x x}+r x V_{x}-r V=0,
$$

where $r$ is the risk-free interest rate and $\sigma$ is the volatility of the underlying asset which are positive constants over the life of the option.

The Black-Scholes equation is an evolution equation; hence it can be solved analytically ${ }^{1-3}$ or numerically ${ }^{4-6}$. However, it appears in the literature that the Black-Scholes model fails to capture the behaviour of option prices under some rare and extreme circumstances. In the past few decades, many authors require the construction of variants of the Black-Scholes models in various financial markets, in which the fractional derivatives are defined. In particular, two types of fractional BlackScholes equations are mainly studied, i.e., timefractional Black-Scholes equation with the Caputo derivatives $^{7,8}$ and pricing-fractional Black-Scholes equation with the Weyl fractional derivative ${ }^{9,10}$.
Furthermore, we will consider a nonlinear timefractional Black-Scholes equation with the nonconstant volatility.

In this paper, we employ the invariant subspace method $^{11}$ to obtain exact solutions of fractional Black-Scholes equations. By assuming the solution to be in the appropriate invariant subspaces, the fractional Black-Scholes equation can easily be reduced to a system of single variable fractional differential equations. After that the Laplace transform method is applied to solve the reduced system of fractional differential equations. The obtained solutions are represented in terms of the MittagLeffler functions, and they approach the solutions of classical Black-Scholes equations with an integer order.

\section{PRELIMINARIES}

We start with some definitions of fractional integrals and derivatives, and then present auxiliary properties.

Definition 1 The left- and right-sided RiemannLiouville fractional integral of order $\alpha$, where $\alpha \in$ $(n-1, n)$ and $n \in \mathbb{N}$, of a function $f$ are given by

$$
\begin{aligned}
& { }_{a} I_{x}^{\alpha} f(x)=\frac{1}{\Gamma(\alpha)} \int_{a}^{x}(x-y)^{\alpha-1} f(y) \mathrm{d} y, \quad x>a, \\
& { }_{x} I_{b}^{\alpha} f(x)=\frac{1}{\Gamma(\alpha)} \int_{x}^{b}(y-x)^{\alpha-1} f(y) \mathrm{d} y, \quad x<b .
\end{aligned}
$$


In particular, the left- and right-sided RiemannLiouville fractional integrals with $a=-\infty$ and $b=\infty$ are often called left- and right-sided Weyl fractional integrals, denoted by ${ }_{-\infty} W_{x}^{\alpha}$ and ${ }_{x} W_{\infty}^{\alpha}$, respectively. Analogously, the left- and right-sided Weyl fractional derivatives are defined, respectively,

$$
\begin{aligned}
{ }_{-\infty} D_{x}^{\alpha} f(x) & =D^{n}\left[{ }_{-\infty} W_{x}^{n-\alpha} f(x)\right], \\
{ }_{x} D_{\infty}^{\alpha} f(x) & =(-1)^{n} D^{n}\left[{ }_{x} W_{\infty}^{n-\alpha} f(x)\right],
\end{aligned}
$$

where $D^{n}=d^{n} / d x^{n}$. The left- and right-sided Caputo fractional derivatives are given by,

$$
\begin{array}{ll}
{ }_{a}^{C} D_{x}^{\alpha} f(x)={ }_{a} I_{x}^{n-\alpha} D^{n} f(x), & x>a, \\
{ }_{x}^{C} D_{b}^{\alpha} f(x)={ }_{x} I_{b}^{n-\alpha} D^{n} f(x), & x<b,
\end{array}
$$

respectively.

Lemma 1 The Weyl fractional derivative of exponential functions ${ }^{12}$ is given by

$$
\begin{aligned}
{ }_{x} D_{\infty}^{\alpha} \mathrm{e}^{-\lambda x} & =\lambda^{\alpha} \mathrm{e}^{-\lambda x}, \\
{ }_{-\infty} D_{x}^{\alpha} \mathrm{e}^{\lambda x} & =\lambda^{\alpha} \mathrm{e}^{\lambda x},
\end{aligned}
$$

where $\operatorname{Re}(\lambda)>0$.

Lemma 2 Laplace transform of the left-sided Caputo derivative of order $\alpha$ where $n-1<\alpha<n$ is

$$
\mathscr{L}\left\{{ }_{a}^{C} D_{x}^{\alpha} f(x)\right\}=s^{\alpha} \mathscr{L}\{f\}(s)-\sum_{k=0}^{n-1} s^{\alpha-k-1} f^{(k)}(0),
$$

where $\operatorname{Re}(s)>0$.

Definition 2 A two parameter function defined by the series expansion

$$
E_{\alpha, \beta}(z)=\sum_{k=0}^{\infty} \frac{z^{k}}{\Gamma(\alpha k+\beta)}, \quad \alpha, \beta, z \in \mathbb{C}, \operatorname{Re}(\beta)>0
$$

is called the Mittag-Leffler function.

Theorem 1 The solution of the linear initial value problem $^{13}$ for $n-1<\alpha<n$

$$
\begin{aligned}
& { }_{a}^{C} D_{t}^{\alpha} y(t)-\lambda y(t)=0, \quad t>0, \\
& y^{(k)}(0)=b_{k}, \quad b_{k} \in \mathbb{R}, \quad k=0,1, \ldots, n-1
\end{aligned}
$$

is given by

$$
y(t)=\sum_{k=0}^{n-1} b_{k} t^{k} E_{\alpha, k+1}\left(\lambda t^{\alpha}\right) .
$$

Lemma 3 The Laplace transform of the MittagLeffler functions is given by

$$
\mathscr{L}\left\{z^{\alpha k+\beta-1} E_{\alpha, \beta}^{(k)}\left( \pm a z^{\alpha}\right)\right\}=\frac{k ! s^{\alpha-\beta}}{\left(s^{\alpha} \mp a\right)^{k+1}},
$$

where $\operatorname{Re}(s)>|a|^{1 / \alpha}$ and $E_{\alpha, \beta}^{(k)}(z)=D^{k} E_{\alpha, \beta}(z)$.

\section{INVARIANT SUBSPACE METHOD}

The method of invariant subspace was initially introduced by Galaktionov ${ }^{11}$ in order to construct particular solutions of evolutionary partial differential equations, and has been extended to classes of fractional partial differential equations ${ }^{14,15}$.

Consider a time-fractional evolution equation,

$$
{ }_{a}^{C} D_{t}^{\alpha} u=\hat{F}[u],
$$

where ${ }_{a}^{C} D_{t}^{\alpha}$ is the left-sided Caputo derivative of order $\alpha$ where $n-1<\alpha<n, n \in \mathbb{N}$. Denote by $\hat{F}[u]=F\left(u, u_{x}, u_{x x}, \ldots, u_{k x}\right)$ the differential operator of order $k$, where $u_{k x}=D^{k} u$. Given a linearly independent functions $\left\{f_{i}(x)\right\}, i=1, \ldots, n$, the $n$ dimensional linear space

$$
W_{n}=\left\langle f_{1}(x), \ldots, f_{n}(x)\right\rangle
$$

is invariant under the operator $\hat{F}$ if $\hat{F}[w] \in W_{n}$ for all $w \in W_{n}$.

The idea of the invariant subspace method ${ }^{11}$ searches for the solution in the invariant subspace in the form

$$
u(x, t)=\sum_{i=1}^{n} \psi_{i}(t) f_{i}(x)
$$

where the coefficients $\psi_{1}(t), \ldots, \psi_{n}(t)$ satisfy the following system of fractional ODEs:

$$
\begin{gathered}
{ }_{a}^{C} D_{t}^{\alpha} \psi_{1}(t)=F_{1}\left(\psi_{1}, \ldots, \psi_{n}\right), \\
\vdots \\
{ }_{a}^{C} D_{t}^{\alpha} \psi_{n}(t)=F_{n}\left(\psi_{1}, \ldots, \psi_{n}\right) .
\end{gathered}
$$

\section{THE TIME-FRACTIONAL BLACK-SCHOLES EQUATIONS}

In this section, we study the time-fractional BlackScholes equation of order $\alpha \in(0,1)$ in the form

$$
{ }_{a}^{C} D_{t}^{\alpha} V+\frac{\sigma^{2}}{2} x^{2} V_{x x}+(r-d) x V_{x}-r V=0,
$$

where $V(x, t)$ is the put or call option price at asset price $x \in \mathbb{R}^{+}$at time $t \in[0, T], T>0, r>0$ is the risk-free interest rate, $\sigma>0$ is the volatility of the underlying asset, and $d$ is the dividend yield. Taking linearly independent functions $1, x^{s-1}, x^{s}$ with $s \in$ $\mathbb{R}$, to form a linear space

$$
W_{1}=\left\langle 1, x^{s-1}, x^{s}\right\rangle .
$$

For linear independent functions 1 and $\ln x$, we define a linear space

$$
W_{2}=\langle 1, \ln x\rangle .
$$


Lemma 4 The partial differential operator

$$
\hat{F}[V]=\frac{\sigma^{2}}{2} x^{2} V_{x x}+(r-d) x V_{x}-r V
$$

admits the invariant subspaces $W_{1}$ and $W_{2}$.

Proof: For any $c_{1}, c_{2}, c_{3} \in \mathbb{R}$,

$$
\hat{F}\left[c_{1}+c_{2} x^{s-1}+c_{3} x^{s}\right]=-r c_{1}+\beta c_{2} x^{s-1}+\gamma c_{3} x^{s},
$$

where $\beta=\frac{1}{2} \sigma^{2}(s-1)(s-2)+(r-d)(s-1)-r$ and $\gamma=\frac{1}{2} \sigma^{2} s(s-1)+(r-d) s-r$, is in $W_{1}$. Thus $W_{1}$ is invariant under the operator $\hat{F}$. Similarly, for any $c_{1}, c_{2} \in \mathbb{R}$,

$$
\hat{F}\left[c_{1}+c_{2} \ln x\right]=\mu-r c_{2} \ln x,
$$

where $\mu=\left(-\frac{1}{2} \sigma^{2}+(r-d)\right) c_{2}-r c_{1}$, which implies that $W_{2}$ is invariant under this operator.

In the following example, by choosing an appropriate invariant subspace, we derive the exact solution of the time-fractional Black-Scholes equation (1) subject to certain terminal conditions, which is the same as that for the classical Black-Scholes equation with an integer derivative order ${ }^{1,3}$.

Example 1 Consider the time-fractional BlackScholes equation (1) subject to the terminal conditions $V(x, T)=g(x)$. We propose the solution of (1) in the form

$$
V(x, t)=\psi_{0}(t)+\psi_{1}(t) x^{s-1}+\psi_{2}(t) x^{s},
$$

where $\psi_{0}, \psi_{1}$, and $\psi_{2}$ are undetermined coefficients. By substitution of (2) into (1), we obtain the time-fractional differential system for the unknown functions $\psi_{0}, \psi_{1}$, and $\psi_{2}$.

$$
\begin{aligned}
& { }_{a}^{C} D_{t}^{\alpha} \psi_{0}(t)=r \psi_{0}(t), \\
& { }_{a}^{C} D_{t}^{\alpha} \psi_{1}(t)=-\beta \psi_{1}(t), \\
& { }_{a}^{C} D_{t}^{\alpha} \psi_{2}(t)=-\gamma \psi_{2}(t) .
\end{aligned}
$$

The solutions of the above system can be obtained by Theorem 1,

$$
\begin{aligned}
& \psi_{0}(t)=\psi_{0}(0) E_{\alpha, 1}\left(r t^{\alpha}\right), \\
& \psi_{1}(t)=\psi_{1}(0) E_{\alpha, 1}\left(-\beta t^{\alpha}\right), \\
& \psi_{2}(t)=\psi_{2}(0) E_{\alpha, 1}\left(-\gamma t^{\alpha}\right) .
\end{aligned}
$$

In particular, the terminal condition is given by $V(x, T)=x^{s}$, which implies that $\psi_{0}(T)=0$, $\psi_{1}(T)=0$, and $\psi_{2}(T)=1$. Since for $0<\alpha<1$, $E_{\alpha, 1}(z)$ has no real zero, we obtain the exact solution

$$
V(x, t)=\frac{E_{\alpha, 1}\left(-\gamma t^{\alpha}\right)}{E_{\alpha, 1}\left(-\gamma T^{\alpha}\right)} x^{s} .
$$

For $\alpha=1$, the exact solution of the classical BlackScholes equation is

$$
V(x, t)=x^{s} \mathrm{e}^{\rho(s)(T-t)},
$$

where $\rho(s)=\left(\frac{1}{2} \sigma^{2} s+r\right)(s-1)-d s$, which coincides with the solution in Ref. 3 .

Example 2 Consider the nonhomogeneous timefractional Black-Scholes equation with $0<\alpha<1$

$$
D_{t}^{\alpha} V+\frac{\sigma^{2} x^{2}}{2} V_{x x}+(r-d) x V_{x}-r V=\ln \sqrt{x}
$$

subject to the terminal condition $V(x, T)=\ln x-1$. In this case, we search for the exact solution of the form

$$
V(x, t)=\psi_{0}(t)+\psi_{1}(t) \ln x,
$$

where the coefficient functions $\psi_{0}(t)$ and $\psi_{1}(t)$ satisfy the system of fractional equations

$$
\begin{aligned}
& D_{t}^{\alpha} \psi_{0}(t)=\left(\frac{\sigma^{2}}{2}-(r-d)\right) \psi_{1}(t)+r \psi_{0}(t), \\
& D_{t}^{\alpha} \psi_{1}(t)=r \psi_{1}(t)+\frac{1}{2} .
\end{aligned}
$$

By applying Theorem 1 to (4), we have the solution

$$
\psi_{1}(t)=\psi_{1}(0) E_{\alpha, 1}\left(r t^{\alpha}\right)+\frac{1}{2} E_{\alpha, \alpha+1}\left(r t^{\alpha}\right) .
$$

It follows from the terminal condition $V(x, T)=$ $\ln x-1$ that $\psi_{0}(T)=-1$ and $\psi_{1}(T)=1$. Hence

$$
\psi_{1}(t)=\rho(\alpha) E_{\alpha, 1}\left(r t^{\alpha}\right)+\frac{1}{2} E_{\alpha, \alpha+1}\left(r t^{\alpha}\right),
$$

where $\rho(\alpha)=\left(2-E_{\alpha, \alpha+1}\left(r T^{\alpha}\right)\right) / 2 E_{\alpha, 1}\left(r T^{\alpha}\right)$. Applying the Laplace transformation to (3) and rearranging the result, we obtain

$$
\begin{aligned}
\mathscr{L}\left\{\psi_{0}\right\} & =\psi_{0}(0) \frac{s^{\alpha-1}}{s^{\alpha}-r}+\left(\frac{\sigma^{2}}{2}-(r-d)\right) \\
& \times \quad\left(\psi_{1}(0) \frac{s^{\alpha-1}}{\left(s^{\alpha}-r\right)^{2}}+\frac{1}{2 s\left(s^{\alpha}-1\right)^{2}}\right) .
\end{aligned}
$$

Taking the inverse Laplace transform yields

$$
\begin{aligned}
& \psi_{0}(t)=\psi_{0}(0) E_{\alpha, 1}\left(r t^{\alpha}\right)+\left(\frac{\sigma^{2}}{2}-(r-d)\right) \\
& \times \quad\left(\psi_{1}(0) t^{\alpha} E_{\alpha, 1}^{\prime}\left(r t^{\alpha}\right)+\frac{t^{2 \alpha}}{2} E_{\alpha, \alpha+1}^{\prime}\left(r t^{\alpha}\right)\right) .
\end{aligned}
$$

Thus the exact solution of the nonhomogeneous time-fractional Black-Scholes equation is given by

$$
\begin{aligned}
& V(x, t)=\psi_{0}(0) E_{\alpha, 1}\left(r t^{\alpha}\right)+\left(\frac{\sigma^{2}}{2}-(r-d)\right) \\
& \times\left(\psi_{1}(0) t^{\alpha} E_{\alpha, 1}^{\prime}\left(r t^{\alpha}\right)+\frac{t^{2 \alpha}}{2} E_{\alpha, \alpha+1}^{\prime}\left(r t^{\alpha}\right)\right) \ln x .
\end{aligned}
$$


Example 3 The time-fractional Black-Scholes equation with variable coefficients

${ }_{a}^{C} D_{t}^{\alpha} u+0.08(2+\sin x)^{2} x^{2} u_{x x}+0.06 x u_{x}-0.06 u=0$,

subjected to the initial condition

$$
u(x, 0)=\max \left\{x-25 \mathrm{e}^{-0.06}, 0\right\},
$$

was studied by using the reconstruction of variation iteration method ${ }^{7}$ and the modified fractional differential transform method ${ }^{8}$. We apply the invariant subspace method using the differential operator

$$
\hat{F}[u]=0.08(2+\sin x)^{2} x^{2} u_{x x}+0.06 x u_{x}-0.06 u,
$$

which admits the invariant subspace $W=\langle 1, x\rangle$, and search for the solution of the form

$$
u(x, t)=\psi_{0}(t)+\psi_{1}(t) x,
$$

where $\psi_{0}(t)$ and $\psi_{1}(t)$ satisfy

$$
\begin{aligned}
& { }_{a}^{C} D_{t}^{\alpha} \psi_{0}(t)=-0.06 \psi_{0}(t), \\
& { }_{a}^{C} D_{t}^{\alpha} \psi_{1}(t)=0 .
\end{aligned}
$$

It follows from the initial condition $u(x, 0)=$ $\max \left\{x-25 \mathrm{e}^{-0.06}, 0\right\}$ that when $u(x, 0)=0$ we obtain the zero solution $u(x, t)=0$, and when $u(x, 0)=x-25 \mathrm{e}^{-0.06}$, the solution is $u(x, t)=$ $-25 \mathrm{e}^{-0.06} E_{\alpha, 1}\left(-0.06 t^{\alpha}\right)+x$. We thus obtain the exact solution,

$$
u(x, t)=\max \left\{x-25 \mathrm{e}^{-0.06} E_{\alpha, 1}\left(-0.06 t^{\alpha}\right), 0\right\},
$$

which is the same as that in Refs. 7,8, but our method is simpler and easier to apply.

\section{THE PRICING-FRACTIONAL BLACK-SCHOLES EQUATION}

Consider the pricing-fractional Black-Scholes equation derived from the finite moment log stable model $^{9}$ with the option price $V(s, t)$ satisfying the modified Black-Scholes equation with the left-sided Weyl-fractional derivative in space,

$$
\begin{aligned}
V_{t}+(r+ & \left.\frac{\sigma^{\alpha}}{2} \sec \frac{\alpha \pi}{2}\right) V_{x} \\
& \quad-\frac{\sigma^{\alpha}}{2} \sec \frac{\alpha \pi}{2}\left({ }_{-\infty} D_{x}^{\alpha} V\right)-r V=0,
\end{aligned}
$$

where $x \in \mathbb{R}^{+}, t \in[0, T], 1<\alpha \leqslant 2$, and the terminal condition $V(x, T)=\Pi(x)$ defined as

$$
\Pi(x)= \begin{cases}\max \left\{\mathrm{e}^{x}-K, 0\right\}, & \text { (European call), } \\ \max \left\{K-\mathrm{e}^{x}, 0\right\}, & \text { (European put). }\end{cases}
$$

By changing the variable $\tau=-\frac{1}{2} \sigma^{\alpha} \sec \frac{1}{2} \alpha \pi(T-t)$, the backward problem is changed into

$$
V_{\tau}=-(\eta-1) V_{x}-\left({ }_{-\infty} D_{x}^{\alpha} V\right)+\eta V
$$

with condition $V(x, 0)=\Pi(x)$, where $\eta=-2 r / \sigma^{\alpha} \sec \frac{1}{2} \alpha \pi$.

Lemma 5 The fractional partial differential operator

$$
\hat{F}[V]=(1-\eta) V_{x}-{ }_{-\infty} D_{x}^{\alpha} V+\eta V
$$

admits the invariant subspace $W=\left\langle\mathrm{e}^{a x}, \mathrm{e}^{b x}\right\rangle$

Proof: For any $c_{1}, c_{2} \in \mathbb{R}$, Lemma 1 gives

$$
\begin{gathered}
\hat{F}\left[c_{1} \mathrm{e}^{a x}+c_{2} \mathrm{e}^{b x}\right]=(1-\eta)\left(c_{1} a \mathrm{e}^{a x}+c_{2} b \mathrm{e}^{b x}\right) \\
-\left(c_{1} a^{\alpha} \mathrm{e}^{a x}+c_{2} b^{\alpha} \mathrm{e}^{b x}\right) \\
+\eta\left(c_{1} \mathrm{e}^{a x}+c_{2} \mathrm{e}^{b x}\right) \\
=\tilde{\beta} c_{1} \mathrm{e}^{a x}+\tilde{\gamma} c_{2} \mathrm{e}^{b x}
\end{gathered}
$$

where $\tilde{\beta}=(1-\eta) a-a^{\alpha}+\eta$ and $\tilde{\gamma}=(1-\eta) b-b^{\alpha}+\eta$, which is in $W$.

We search for the solution of the pricingfractional Black-Scholes equation (7) of the form

$$
V(x, \tau)=\psi_{1}(\tau) \mathrm{e}^{a x}+\psi_{2}(\tau) \mathrm{e}^{b x},
$$

where $\psi_{1}$ and $\psi_{2}$ are described by the ODEs

$$
\frac{\mathrm{d} \psi_{1}}{\mathrm{~d} \tau}=\tilde{\beta} \psi_{1}, \quad \frac{\mathrm{d} \psi_{2}}{\mathrm{~d} \tau}=\tilde{\gamma} \psi_{2} .
$$

The above equations are solved for $\psi_{1}(t)$ and $\psi_{2}(t)$ to obtain a particular exact solution of (7) as

$$
\begin{aligned}
V(x, \tau)= & \psi_{1}(0) \mathrm{e}^{\tilde{\beta} \tau+a x}+\psi_{2}(0) \mathrm{e}^{\tilde{\gamma} \tau+b x} \\
= & \psi_{1}(0) \mathrm{e}^{\left(a(1-\eta)-a^{\alpha}+\eta\right) \tau+a x} \\
& +\psi_{2}(0) \mathrm{e}^{\left(b(1-\eta)-b^{\alpha}+\eta\right) \tau+b x} .
\end{aligned}
$$

Remark 1 Let $V_{\mathrm{p}}(x, \tau)$ and $V_{c}(x, \tau)$ denote the value of the European put and call options, respectively. The initial condition for the put and call options (6) gives $a=0, b=1, \psi_{1}(0)=K$, and $\psi_{2}(0)=-1$ for the put option, and $a=0, b=1$, $\psi_{1}(0)=-K$, and $\psi_{2}(0)=-1$ for the call option, which produces the same result as in Ref. 10, and the finite moment log stable model demonstrated in (5) satisfies the put-call parity,

$$
V_{c}(x, \tau)-V_{\mathrm{p}}(x, \tau)=\mathrm{e}^{x}-K \mathrm{e}^{-\eta \tau} .
$$




\section{THE NONLINEAR FRACTIONAL BLACK-SCHOLES EQUATION}

In this section, the nonlinear fractional BlackScholes equation with a nonconstant volatility is considered. It was proposed in Ref. 4 for integer order $\alpha=1$ and was referred to as the risk adjusted pricing method model ${ }^{5}$. In this study $\sigma\left(x, t, V, V_{x}, V_{x x}\right)$ is chosen in the form

$$
\sigma^{2}=\tilde{\sigma}^{2}\left(1-3\left(\frac{C^{2} M}{2 \pi} x V_{x x}\right)^{1 / 3}\right),
$$

where $M$ and $C$ are nonnegative constants representing cost measure and the risk premium measure, respectively.

By replacing the constant volatility $\sigma$ in (1) with the nonconstant volatility in (8), we obtain the nonlinear time-fractional Black-Scholes equation with $0<\alpha<1$,

$D_{t}^{\alpha} V+\frac{\tilde{\sigma}^{2}}{2} x^{2} V_{x x}-\tilde{\sigma}^{2} \mu x\left(x V_{x x}\right)^{4 / 3}+r x V_{x}-r V=0$,

where $\mu=\frac{3}{2}\left(C^{2} M / 2 \pi\right)^{1 / 3}$.

Lemma 6 The nonlinear partial differential operator

$$
\hat{F}[V]=\frac{\tilde{\sigma}^{2}}{2} x^{2} V_{x x}-\tilde{\sigma}^{2} \mu x\left(x V_{x x}\right)^{4 / 3}+r x V_{x}-r V
$$

admits the invariant subspace $W=\langle 1, x, x \ln x\rangle$.

Proof: For any $c_{1}, c_{2}, c_{3} \in \mathbb{R}$, we have

$$
\begin{aligned}
\hat{F}\left[c_{1}+\right. & \left.c_{2} x+c_{3} x \ln x\right]=\frac{\tilde{\sigma}^{2}}{2} x^{2}\left(\frac{c_{3}}{x}\right)-\tilde{\sigma}^{2} \mu x c_{3}^{4 / 3} \\
& +r x\left[(1+\ln x) c_{3}+c_{2}\right] \\
& \quad-r\left[c_{1}+c_{2} x+c_{3} x \ln x\right] \\
=- & r c_{1}+\left[\left(\frac{\tilde{\sigma}^{2}}{2}+r\right) c_{3}-\tilde{\sigma}^{2} \mu c_{3}^{4 / 3}\right] x,
\end{aligned}
$$

which is in $W$ to complete the proof.

Example 4 Consider the nonlinear time-fractional Black-Scholes equation (9) with a solution in the form

$$
V(x, t)=\psi_{0}(t)+\psi_{1}(t) x+\psi_{2}(t) x \ln x,
$$

where $\psi_{0}(t), \psi_{1}(t)$, and $\psi_{2}(t)$ are described by the system of fractional differential equations

$$
\begin{aligned}
& D_{t}^{\alpha} \psi_{0}(t)=r \psi_{0}(t) \\
& D_{t}^{\alpha} \psi_{1}(t)=-\left(\frac{\tilde{\sigma}^{2}}{2}+r\right) \psi_{2}(t)-\tilde{\sigma}^{2} \mu\left(\psi_{2}(t)\right)^{4 / 3} \\
& D_{t}^{\alpha} \psi_{2}(t)=0
\end{aligned}
$$

Solving equations (10) and (12) gives $\psi_{0}(t)=$ $\psi_{0}(0) E_{\alpha}\left(r t^{\alpha}\right)$ and $\psi_{2}(t)=c, c \in \mathbb{R}$. Substituting $\psi_{2}(t)=c$ in (11) yields

$$
D_{t}^{\alpha} \psi_{1}(t)=-\left(\frac{\tilde{\sigma}^{2}}{2}+r\right) c-\tilde{\sigma}^{2} \mu c^{4 / 3},
$$

which has the solution

$\psi_{1}(t)=\psi_{1}(0)-\frac{1}{\Gamma(\alpha+1)}\left(\left(\frac{\tilde{\sigma}^{2}}{2}+r\right) c+\tilde{\sigma}^{2} \mu c^{4 / 3}\right) t^{\alpha}$.

Thus the solution of (9) is

$$
\begin{aligned}
& u(x, t)=\psi_{0}(0) E_{\alpha}\left(r t^{\alpha}\right)+c x \ln x \\
& \quad+\left[\psi_{1}(0)-\frac{t^{\alpha}}{\Gamma(\alpha+1)}\left(r c+\left(\frac{c}{2}+\mu c^{4 / 3}\right) \tilde{\sigma}^{2}\right)\right] x
\end{aligned}
$$

\section{CONCLUSIONS}

In this study, exact solutions of linear and nonlinear fractional Black-Scholes equations are in invariant subspace as described in Ref. 11. We have shown that fractional Black-Scholes equations admit certain invariant subspaces, which are used to derive exact solutions to various terminal conditions for fractional Black-Scholes equations. Several examples are demonstrated to illustrate the efficiency of the invariant subspace method for constructing solutions comparing to former studies in literature.

\section{REFERENCES}

1. Jódar L, Sevilla-Peris P, Cortés JC, Sala R (2005) A new direct method for solving the Black-Scholes equation. Appl Math Lett 18, 29-32.

2. Rodrigo MR, Mamon RS (2006) An alternative approach to solving the Black-Scholes equation with time-varying parameters. Appl Math Lett 19, 398-402.

3. Bohner M, Zheng Y (2009) On analytical solutions of the Black-Scholes equation. Appl Math Lett 22, 309-13.

4. Jandačka M, Ševčovič D (2005) On the risk-adjusted pricing-methodology-based valuation of vanilla options and explanation of the volatility smile. $J \mathrm{Appl}$ Math 2005, 235-58.

5. Ankudinova J, Ehrhardt M (2008) On the numerical solution of nonlinear Black-Scholes equations. Comput Math Appl 56, 799-812.

6. Ahn J, Kang S, Kwon YH (2010) A Laplace transform finite difference method for the Black-Scholes equation. Math Comput Model 51, 247-55.

7. Akrami MH, Erjaee GH (2015) Examples of analytical solutions by means of Mittag-Leffler function of fractional Black-Scholes option pricing equation. Fract Calc Appl Anal 18, 38-47. 
8. Ravi Kanth ASV, Aruna K (2016) Solution of time fractional Black-Scholes European option pricing equation arising in financial market. Nonlinear Eng 5, 269-76.

9. Cartea A, del-Castillo-Negrete D (2007) Fractional diffusion models of option prices in markets with jumps. Physica A 374, 749-63.

10. Chen W, Xu X, Zhu SP (2014) Analytically pricing European-style options under the modified BlackScholes equation with a spatial-fractional derivative. Quart Appl Math 72, 597-611.

11. Galaktionov VA, Svirshchevskii SR (2007) Exact Solutions and Invariant Subspaces of Nonlinear Partial Differential Equations in Mechanics and Physics, Chapman \& Hall/CRC, Boca Raton, FL.

12. Hanneken JW, Vaught DM, Achar BNN (2007) Enumeration of the real zeros of the Mittag-Leffler function $E_{\alpha}(z), 1<\alpha<2$. In: Sabatier J, Agrawal OP, Machado JAT (eds) Advances in Fractional Calculus, Springer, Dordrecht, pp 15-26.

13. Ishteva M (2005) Properties and applications of the Caputo fractional operator. MSc thesis, Universität Karlsruhe.

14. Gazizov RK, Kasatkin AA (2013) Construction of exact solutions for fractional order differential equations by the invariant subspace method. Comput Math Appl 66, 576-84.

15. Choudhary S, Daftardar-Gejji V (2017) Invariant subspace method: A tool for solving fractional partial differential equations. Fract Calc Appl Anal 20, 477-93. 